\title{
Using circular dot pattern code tag for medical information on the round type medical package
}

\author{
Jae Youn Shim \\ Department of Computer Engineering, \\ Kyungdong University, \\ 46 Bongpo4-gil, Gosung, \\ Gangwondo 24764, Republic of Korea \\ Email: simpo@kduniv.ac.kr

\section{Seong-Whan Kim*} \\ School of Computer Science, \\ University of Seoul, \\ 163 Seoul Siripdae-ro, \\ Dongdaemoon-gu, Republic of Korea \\ Email:swkim7@uos.ac.kr \\ ${ }^{*}$ Corresponding author
}

\begin{abstract}
Administration of medicines is important issue for patient safety in medical applications. Especially, manufacture date and expiry date are very important. Medical applications have strong needs for embedded tags on medical bottles to facilitate medicine management. One-dimensional (1D) barcode cannot represent the required information capacity. Two-dimensional (2D) barcode and radio-frequency identification (RFID) systems can support information capacity. However, 2D barcode is hard to recognition, if $2 \mathrm{D}$ barcode tag is attached on curvature surface. RFID is 100 times more expensive than barcode systems and also it is difficult to embed RFID tags on curvature. In this paper, we propose circular dot pattern code (CDPC) tag for cylindrical and round type medical bottle identification and management. CDPC is based on topology of dot pattern code, and it is well suited to recognising tags in curvature environments. Experimental results show that CDPC outperformed 2D barcode in its recognition rate on curvature, including cylindrical and round shape.
\end{abstract}

Keywords: identification; medical package; code recognition system; dot pattern code; pattern recognition; circular tag; RFID; radio-frequency identification; QR; quick response code; information embedding; topology; ECC; error correction code.

Reference to this paper should be made as follows: Shim, J.Y. and Kim, S-W. (2016) 'Using circular dot pattern code tag for medical information on the round type medical package', Int. J. Advanced Media and Communication, Vol. 6, No. 1, pp.48-56.

Biographical notes: Jae Youn Shim received his $\mathrm{PhD}$ from the University of Seoul, South Korea in 2015. He is currently an Assistant Professor at the Department of Computer Engineering, Kyungdong University, South Korea. His research interests include coding theory, image analysis for video forensics, ubiquitous computing, computer vision, and Big data based data mining. 
Seong-Whan Kim received his PhD in EECS from KAIST, Taejon in 1999. He worked for IMT-2000 infrastructure division as a software team leader in LG Electronics from 1997 to 2000, and was responsible for wireless LAN infrastructure design as a Senior Software Engineer in Cisco Systems Inc. San Jose from 2000 to 2001. He is currently a Full Professor at the School of Computer Science, University of Seoul, South Korea. Since 2015, he has worked for the Ministry of Culture, Sports and Tourism as a National R\&D Director. His research interests include mobile communication systems, mathematical modelling of human visual system and its application to multimedia coding/restoration/transmission.

This paper is a revised and expanded version of a paper entitled 'Smart tag for identifying vials in medical applications using circular dot pattern code (CDPC) with curvature robustness' presented at 2015 International Conference on Platform Technology and Service (PlatCon-15), International Convention Center Jeju (ICC JEJU), Korea, 26-28 January, 2015.

\section{Introduction}

In medical applications, taking the right medicine is an important for the patient's health. Abuse of the medicine may be harmful to the health of the patient. The pharmaceutical packaging market is constantly advancing and has experienced annual growth of at least $5 \%$ per annum in the past few years. According to Jain et al. (2008), the pharmaceutical packaging market was reckoned to be worth over $\$ 20$ billion a year in 2008. The pharmaceutical packaging product types include prescription bottles, vials, ampoules and syringes with carton. Prescription bottles contain medicine prescribed by physicians. A vial is one of prescription bottles and a small glass or plastic bottle. An ampoule is a small sealed vial which is used to contain and preserve a sample. A syringe is a pump consisting of a plunger that fits tightly in a tube. A carton is a box or container usually made of paperboard and sometimes of corrugated fibreboard. Most pharmaceutical packages are supplied packed in cartons. Packaging is defined as the collection of different components that surround the pharmaceutical product from the time of production until its use.

Medicine information of manufacture date and expiry date are an important for patient safety. Korean Government enacted, specialty pharmaceuticals attached two-dimensional (2D) barcodes or radio-frequency identification (RFID) in 2013. Thing like prescription bottles need its information of manufacture date and expiry date for user safety. 2D barcode like QR code system is difficult to recognition on cylindrical and round type surface. RFID has a large capacity of information, but it is 100 times more expensive than barcode systems, and vulnerable to impact. If tag code system is recognised on cylindrical type bottle, it needs transformation correction. We perform topological graph analysis to determine the topological distance between dot pixels.

In this paper, we propose cylindrical and round type medical bottles tagging using circular dot pattern code (CDPC). The rest of this paper is organised as follows. Section 2 reviews related works on barcode, QR code, RFID and CDPC. Section 3 shows the experimental results of cylindrical and round type bottle tag using CDPC. We conclude in Section 4. 


\section{Related works}

In information systems, a tag is a non-hierarchical keyword or term assigned to a piece of information. This kind of metadata helps describe an item and allows it to be found again by browsing or searching. Tags are generally chosen informally and personally by the item's creator or by its viewer, depending on the system. Tag should be able to recognise, even if the tag is attached to the curved surface for cylindrical and round type medical bottles. Figure 1 shows shape of a prescription bottle, a vial, an ampoule and a syringe. Prescription bottles have various shape type include cylindrical/round, square, rectangular and oval. A vial is one of prescription bottles and a small glass or plastic bottle, often used to store medication as liquids, powders or capsules. An ampoule is a small sealed vial which is used to contain and preserve a sample, usually a solid or liquid. A syringe is a pump consisting of a plunger that fits tightly in a tube. The plunger can be pulled and pushed along inside a cylindrical tube. Their shape type is cylindrical and round.

Figure 1 Prescription bottle, vial, ampoule, and syringe (see online version for colours)
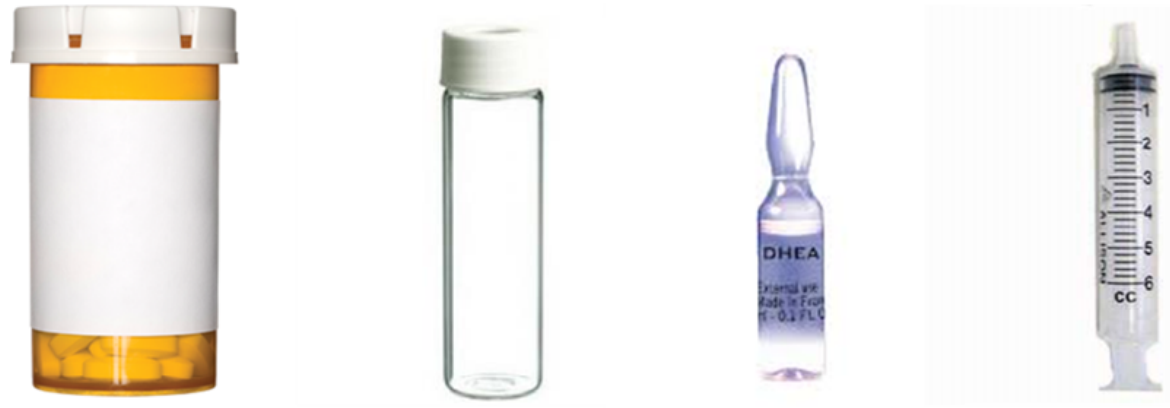

One-dimensional (1D) barcodes have been developed and used for distribution in fields such as industry and the military. 1D barcode is composed of patterns such as guard bars that indicate the initial, final and central positions of the image code and data patterns that encode the numeric data and checksum digit (Pavlidis et al., 1990). 1D bar code patterns are expressed in terms of relative thickness ratios of parallel black and white bars. The mapping between messages and barcodes is called a symbology. The specification of a symbology includes the encoding of the single digits/characters of the message as well as the start and stop markers into bars and space, the size of the quiet zone required to be before and after the barcode as well as the computation of a checksum. The quiet zone should be at least 10 times the width of the narrowest bar/space element. It is mandatory at the left- and right-hand side of the barcode. Start/stop character must be the first and last character appearing in the complete barcode. Inter character caps are the space between characters. Thus, by sampling the pixels on scan lines placed across the patterns, the decoder can recognise the code if at least one scans line covers all the patterns. After reading all the patterns, the decoder verifies the result by using the checksum digit in the data patterns.

QR code is most popular 2D barcode. QR codes (ISO, 2000), 2D barcodes created by Denso-Wave, are the most popular type of $2 \mathrm{D}$ codes. They are currently used in broader contexts, and their use is increasing to include convenience-oriented applications for 
mobile phone users. According to Denso Wave Inc. (2014), QR codes storing addresses and URLs may appear in various advertisements, business cards, or any object that would allow a user to obtain information on a particular item. QR codes are capable of handling various types of data such as numeric and alphabetic characters, Japanese characters, symbols, and binary and control codes. Forty versions of QR code symbols are available, and up to 7089 characters can be encoded in each symbol. Version 1 specifies $21 \times 21$ modules. Each version of the symbol adds four modules on each side until reaching version 40, which contains $177 \times 177$ modules. Moreover, QR codes have error correction capability. Even in the event of a symbol partially obscured by dirt or damage. QR codes can correct errors in $\sim 7 \%, 15 \%, 25 \%$ and $30 \%$ of code words at levels L, M, Q and $\mathrm{H}$, respectively. Finder pattern is pattern for detecting the position of the QR Code. By arranging this pattern at the three corners of a symbol, the position, the size and the angle of the symbol can be detected. This finder pattern consists of a structure that can be detected in all directions. Alignment pattern is a pattern for correcting the distortion of the QR Code. It is highly effective for correcting non-linear distortions. The central coordinate of the alignment pattern will be identified to correct the distortion of the symbol. For this purpose, a black isolated cell is placed in the alignment pattern to make it easier to detect the central coordinate of the alignment pattern. Timing pattern is a pattern for identifying the central coordinate of each cell in the QR Code with black and white patterns arranged alternately. It is used for correcting the central coordinate of the data cell when the symbol is distorted or when there is an error for the cell pitch. It is arranged in both vertical and horizontal directions. 2D bar code system is used in various fields. Wang et al. (2016) proposed a scheme of embedding 2D barcodes into given Visual Cryptography shares to prevent cheating.

Radio-frequency identification (RFID) is a technology that uses radio waves communication to exchange data between a reader and an electronic tag attached to an object, for the purpose of identification and tracking. The RFID has high potential such as supply chain management, access control with identificatiFigon card, and asset tracking system (Finkenzeller, 2003). RFID system is composed of a reader and tags, where RF reader simply reads and writes data from each entity (RF tag). RFID reader also supplies energy for tags using radio frequency, requests information on tags using tag ID, and interprets received signal from an ID-specified tag. RFID tag responds to reader and it has unique identification information in tag ID. RFID have been studied for various applications. Earlier studies (Han et al., 2015) showed RFID tag embedded in 2D barcode on PCB.

Circular dot pattern code recognition scheme is on the basis of topological matching. CDPC recognition scheme includes the following components:

1 image capture with camera

2 dot-pattern detection using Harris corner detector

3 template search

4 projective transformation correction

5 data extraction (Shim and Kim, 2014).

Figure 2 shows overall structure of CDPC. 
Figure 2 Overall structure of CDPC

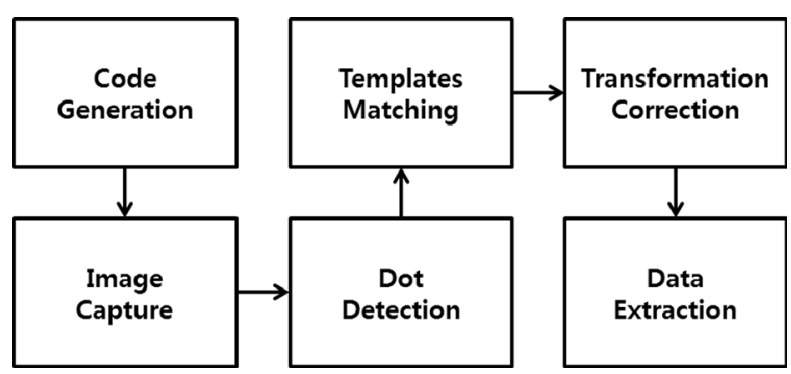

\section{Design}

In medical applications, administration of medicines is important issue for patient safety. Medical applications have strong needs for embedded tags in vials to facilitate medicine management. Tag should be able to recognise, even if the tag is attached to the curved surface for cylindrical and round type prescription bottles. Figure 3 shows how code transformation made on cylinder type object with camera. The width of the code area is up to $2 / \pi$ times smaller emerges on cylinder type.

Figure 3 Transformation on cylinder type object with camera

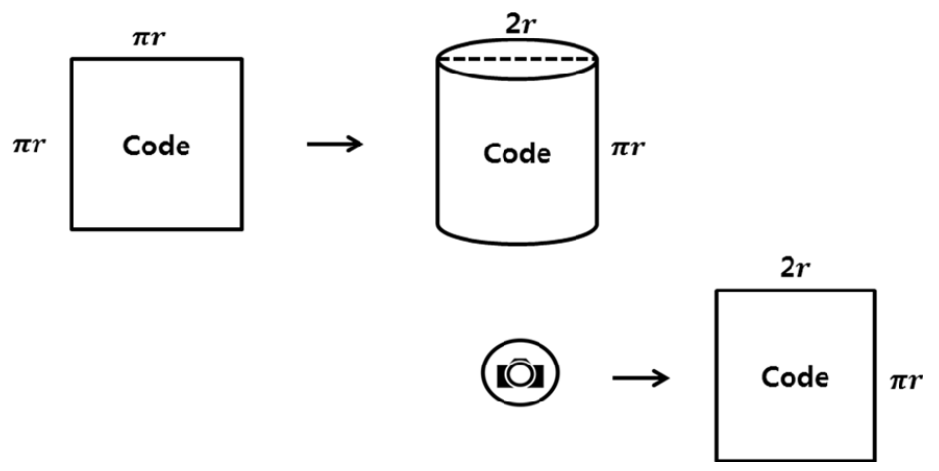

We propose tag using topology-based dot code system. CDPC is on the basis of topological matching. Topology is a major area of mathematics concerned with properties that are preserved under continuous deformations of objects, such as deformations that involve stretching, but no tearing or gluing. It emerged through the development of concepts from geometry and set theory, such as space, dimension and transformation. A set $X$ along with a collection $T$ of subsets of it is said to be a topology if the subsets in $T$ obey the following properties:

- the (trivial) subsets $X$ and the empty set are in $T$

- whenever sets $A$ and $B$ are in $T$, then so is their intersection

- whenever two or more sets are in $T$, then so is their union. 
In topology and related areas of mathematics, a neighbourhood is one of the basic concepts in a topological space. Intuitively speaking, a neighbourhood of a point is a set containing the point where you can move that point some amount without leaving the set (Steen and Seebach, 1978).

Two continuous map $f, g: X \rightarrow Y$ are called homotopic $(f \sim g)$. If $f$ can be continuously deformed into $g$ in the class of continuous maps, if there is a one-parameter family of continuous map beginning with $f$ and ending with $g$ (Viktor, 2001). If $k: X \rightarrow Y$ is continuous map, and if $F$ is a path homotopy in $X$ between the paths $f$ and $f^{\prime}$, then $k \circ F$ is a path homotopy in $Y$ between the paths $k \circ f$ and $k \circ f$ in Figure 4 (James, 2000).

Figure 4 Homotopy

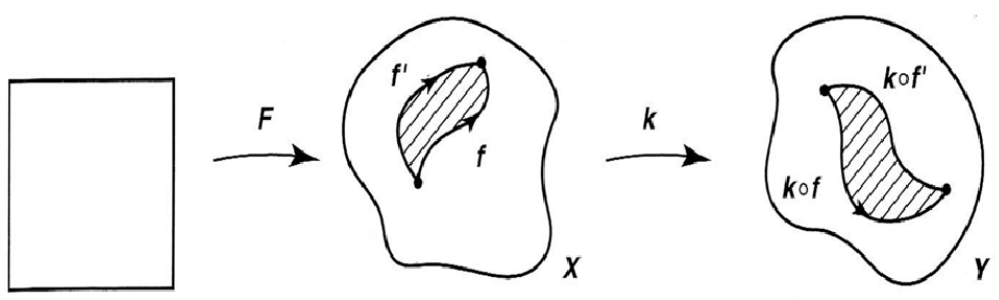

CDPC recognition system includes the following components: First, camera capture code image include low-cost and low-quality camera. Secondly, system detects code information dot using filtering and graph mapping, and matches the template using Depth First Search. Image transformation corrects using topology-based techniques. Data are extracted using this information and decoding process-based Reed-Solomon code. Figure 5 shows diagram of three circle data type of CDPC.

Figure 5 Structure of three circle type CDPC (see online version for colours)

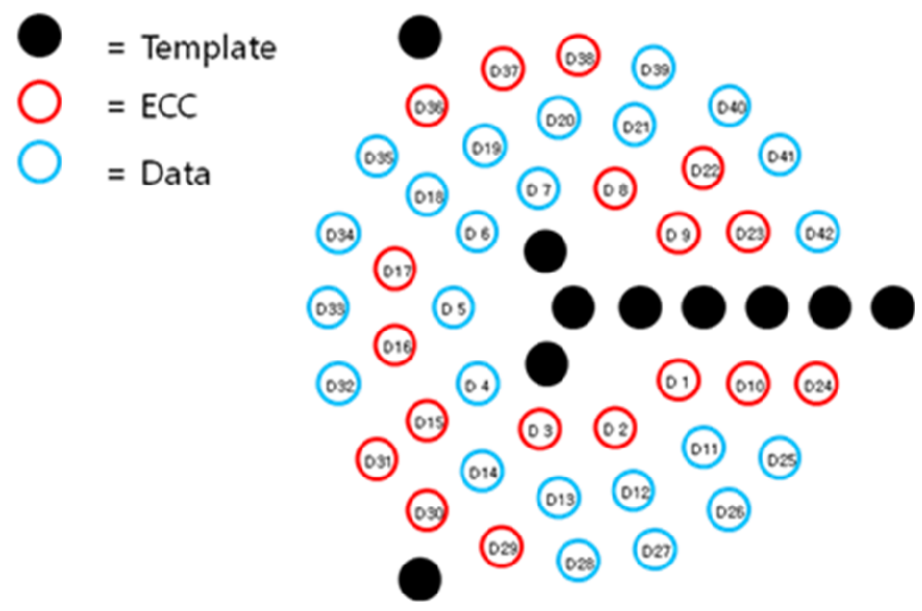

We set the centred line as a template for projective transformation correction. There are centred point and sine and cosine cost is zero of each circle. Templates help in detecting and recognising image rotation and in determining the skew from the camera 
projection. The centred line template and side two points on the three divide of circle were used to determine the lining. This three circle data type CDPC can be inserted 42 binary bits.

Several problems are associated with the detection of pattern code. Geometric distortion can occur, depending on the scanning code location. CDPC proposed scheme for correction of transformation that is detected dot location include data and CDPC use this information for creating the graph and templates searching for correction of the location information. System extract of data using projective transformation-based templates location information. First, we make graph using dot detection and Secondly, we search the six dot templates include circle centred dot point using graph information. Figure 6 shows code image transformation and maintaining of templates relationships. We use the relationship between the points is maintained in the shape transformation for CDPC recognition system. We search template and find transformation function. We use homotopic inverse function for image restoration.

Figure 6 Invariant templates relationships under CDPC transformation (see online version for colours)
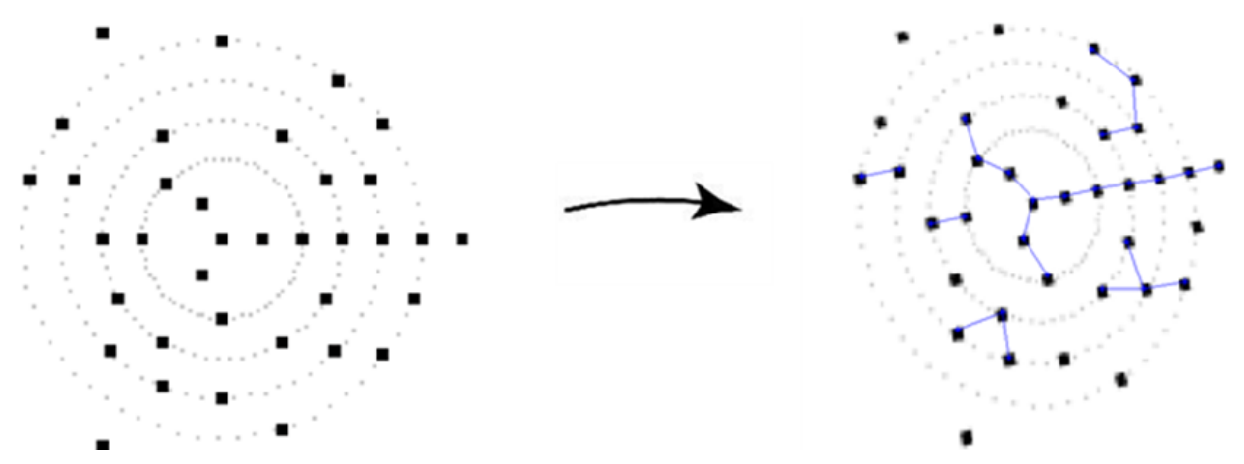

\section{Experimental results}

In this paper, major feature of the system is cylindrical and round type prescription bottles tag. We test the system performance for code pattern recognition on cylinder type object. Cylinder object is tagged QR codes and CDPC, and we test recognition. Figure 7 shows QR code tagging on cylinder object. QR code cannot work well on curved surface, because QR code is hard to recognise under cylinder type distortion. Figure 8 shows CDPC tagging with cylinder object and CDPC can work well because CDPC recognition is basically topological dot matching which is robust under cylinder type of distortion.

Figure 9 shows QR code and CDPC recognition result on cylinder object. The code width transform between $1: 1-1: 2 / \pi$ reduction rate with 0.01 intervals. Experiment was repeated 100 times for each interval. CDPC has good recognition result before $0.8 \mathrm{CDPC}$ neighbourhood relationship is destroyed after $0.8 . X$-axis is width reduction rate and $Y$-axis is code recognition rate. 
Figure 7 Recognition test of QR code on cylinder type object (see online version for colours)

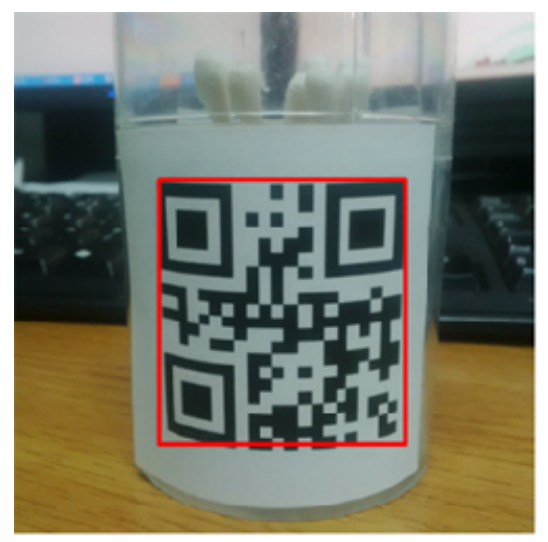

Figure 8 Recognition test of CDPC on cylinder type object (see online version for colours)
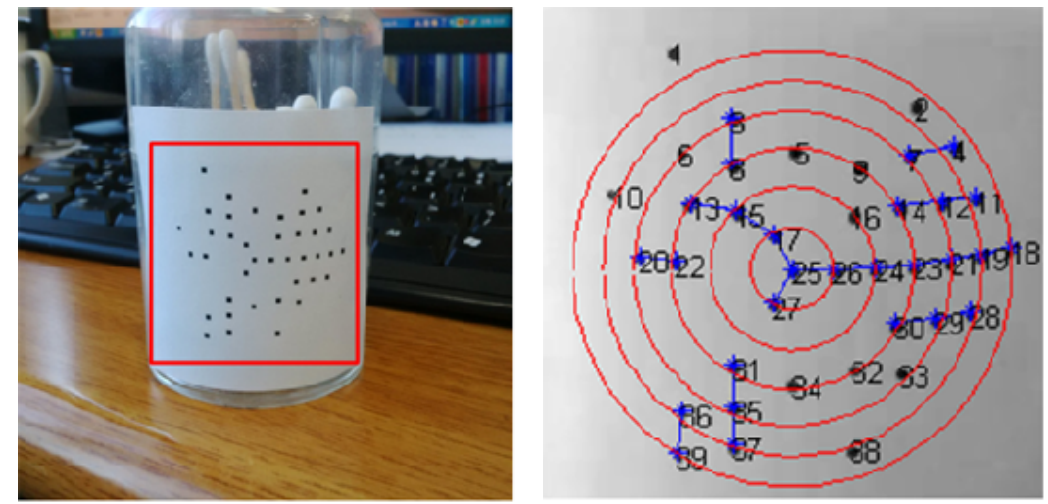

Figure 9 Experimental results of QR and CDPC on cylinder object (see online version for colours)

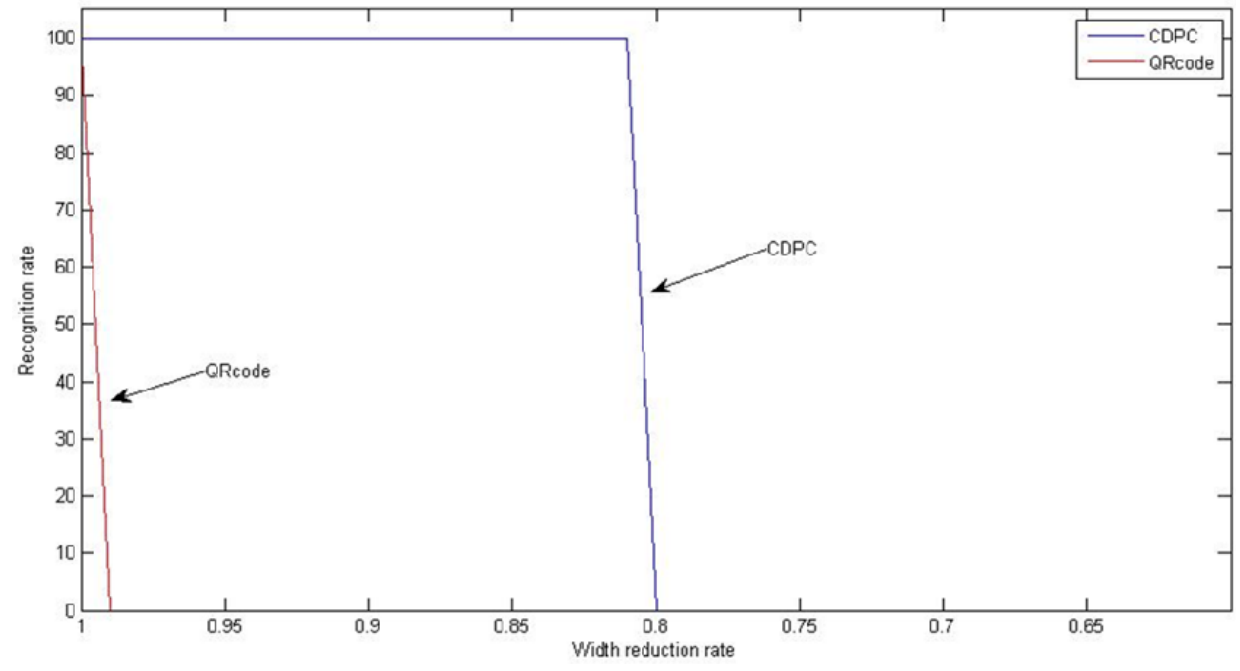




\section{Conclusions}

Medical applications have requirements of embedded tags on medical bottles to facilitate secure medicine management and user safety. 2D barcode like square-based QR code is hard to recognition on cylindrical and round type prescription bottles, and RFID can easily break or damage on cylindrical and round type bottles. We had experiments for tag recognition on cylindrical type bottles with QR code and CDPC. Topology-based CDPC recognition system has good performance on cylindrical and round type prescription bottles. Experimental results show that CDPC outperformed 2D barcodes in its recognition rate on cylinder object. Our future work is to develop CDPC scheme, which embeds information on $3 \mathrm{D}$ printing products for $3 \mathrm{D}$ object identification and management.

\section{Acknowledgements}

This work was supported by the 2014 Research Fund of the University of Seoul.

\section{References}

Denso Wave Inc. (2014) QRcode.com - Many Ways of using QR Codes, http://www.qrcode.com/ en/about/howtouse.html (Accessed 15 February, 2015).

Finkenzeller, K. (2003) RFID Handbook: Fundamentals and Application in Contact-Less Smart Card and Identification, 2nd ed., John Wiley \& Sons Ltd, Chichester, UK.

Han, J., Wang, G. and Sidén, J. (2015) 'Fragment-type UHF RFID tag embedded in QR barcode label', Electronics Letters, Vol. 51, No. 4, pp.313-315.

International Organization for Standardization (ISO) (2000) Information Technology - Automatic Identification and Data Capture Techniques - Bar Code Symbology - QR Code, ISO/IEC 18004.

Jain, U.K., Goupale, D.C. and Nayak, S. (2008) Pharmaceutical Packaging Technology, 1st ed., PharmaMed Press. Hyderabad, India.

James, R.M. (2000) Topology, 2nd ed., Prentice-Hall Inc., Upper Saddle River, New Jersey, USA.

Pavlidis, T., Swartz, J. and Wang, Y.P. (1990) 'Fundamentals of bar code information theory', IEEE Computer, IEEE Computer Society, Vol. 23, No. 4, pp.74-86.

Shim, J-Y. and Kim, S-W. (2014) 'Design of circular dot pattern code (CDPC) for maximum information capacity and robustness on geometric distortion/noise', Multimedia Tools and Applications, Vol. 70, No. 3, pp.1941-1955.

Steen, L.A. and Seebach, A.J. (1978) Counterexamples in Topology, Springer, Berlin.

Viktor, A.V. (2001) Introduction to Topology, American Mathematical Society, Providence, Rhode Island, USA.

Wang, G., Liu, F. and Yan, W.Q. (2016) '2D barcodes for visual cryptography', Multimedia Tools and Applications, Vol. 75, No. 2, pp.1223-1241. 